\title{
Optical design, modelling and tolerancing of a Fizeau interferometer dedicated to astrometry
}

\author{
S. Loiseau ${ }^{1}$ and S. Shaklan ${ }^{2}$ \\ 1 DESPA, Observatoire de Paris, F-92195 Meudon Cedex, France \\ 2 Jet Propulsion Laboratory, California Institute of Technology, 4800 Oak Grove Drive, Pasadena, California 91109, \\ U.S.A.
}

Received September 19; accepted November 2, 1995

\begin{abstract}
Space-based interferometers dedicated to wide-angle astrometry would dramatically increase the accuracy of angular measurements fundamental to a wide range of astrophysical problems. The proposed Global Astrometric Interferometer for Astrophysics (GAIA), a continuously rotating instrument comprising two or three interferometers, will reach the $5-20 \mu$ as level on more than 35 million objects. The necessary wide field-of-view for such a precision could be obtained with a Fizeau interferometer. We designed and modelled a $2.6 \mathrm{~m}$ baseline interferometer with two $40 \mathrm{~cm}$ apertures, and overall dimensions compatible with the size of the Ariane V payload shroud. It has a $\sim 1$ degree diffraction limited field-of-view. The response of the optical system to small perturbations on each optical element is given in terms of fringe visibility, which is shown to depend mainly on sub-aperture spot separation. The robustness of the design to thermal, mechanical and manufacturing errors is discussed.
\end{abstract}

Key words: instrumentation: interferometers — telescopes — astrometry

\section{Introduction}

Astronomical interferometry has now passed the purely demonstrative stage and has made important scientific contributions (Beckers \& Merkle 1991) including the field of astrometry (Shao et al. 1987). Several ground-based interferometers are in operation, measuring star diameters or searching for extra-solar planets for instance. Sending an interferometer to space raises new and challenging problems, such as fitting the instrument in a confined payload shroud or controlling its stability to various perturbations. This paper presents the study of an astrometryoriented interferometer of the Fizeau type. The interferometer is modelled using a compact three-mirror telescope specifically designed to fit in the payload envelope of the Ariane V launcher and which is presented in the first part of Sect. 2. The operational mode of the interferometer and its associated formalism, as well as the method used to estimate the visibility of the interference fringes is presented in the second part of the same section. The Fizeau interferometer is then analyzed in its whole and tolerances to mechanical perturbations, due for instance to thermal variations, are given (Sect. 3). We present a unified curve of tolerancing giving the fringe visibility as a function of

Send offprint requests to: S. Loiseau

(loiseau@bluenote.obspm.fr) the magnitude and type of the applied perturbation. It is shown that as the interferometer is mechanically perturbed (e.g. tilt or displacement of any optical element), the fringe contrast drops mainly as a function of spot separation in the image plane, with aberrations playing only a secondary role. The optical aberrations of the instrument are small and a detailed analysis of the distortion is also given in Sect. 3.

The simplest astronomical interferometer is a mirror covered by a mask in which two apertures are pierced. Named after Fizeau, this kind of interferometer differs from the so-called Michelson interferometer by the homothetical relationship between its entrance and exit pupils. Essentially, the entrance pupil of a Fizeau interferometer is composed of two or more mirrors which are all part of a giant virtual primary mirror, whereas a Michelson interferometer comprises independent light collectors feeding a common beam combiner. For our application, namely wide field astrometry, the Fizeau configuration is required because it offers a very much wider field-of-view than a Michelson of the same entrance pupil configuration.

The strict performance requirements on these future instruments lead to severe stability constraints on the optical and thus mechanical structure. For instance, achieving an astrometric accuracy of 20 micro-arcsecond ( $\mu$ as) using a $3 \mathrm{~m}$ baseline requires a measurement 
accuracy in Optical Path Difference (OPD) between the two interfering beams of 300 picometers. Moreover, this OPD must be stabilized so as to remain smaller than a fraction of the coherence length $\lambda^{2} / \Delta \lambda$. In the case of a Fizeau interferometer, the Airy disks formed by the two apertures must add coherently on the focal plane, imposing stability constraints on the optical train. Achieving these requirements may be even more challenging in space.

There are several proposed and well-studied spacebased interferometers (Shao 1993; Reasenberg et al. 1994). Space provides not only its traditional advantages absence of atmospheric turbulence, access to the whole optical spectrum - but is also the only possible platform for global astrometry, which consists in performing a global reduction of the data over the whole celestial sphere, so as to eventually obtain a coherent reference frame. Interferometers seem to be particularly well fitted for astrometry since they enable an accurate control of systematic errors, which is precisely the key to high-precision astrometric measurements. Furthermore, interferometers have the intrinsic capability to decouple sensitivity (i.e. the lightgathering power) and resolution, which makes it possible to reach higher resolutions than those possible with equivalent conventional telescopes.

The scientific case for very accurate astrometry is compelling. For example, stellar luminosities estimates require the knowledge of stellar distances, which can only be directly determined from measurements of trigonometric parallaxes. Another example is the dramatic improvement of the knowledge of the extra-galactic distance scale that would result from the measurements of the distances of hundreds of Cepheids and RR Lyrae stars at the $20 \mu$ as level. Parallaxes at the $50 \mu$ as level for $\mathrm{K}$ giants with a broad range of metallicities would considerably improve the accuracy of the calculation of the surface density of the Galactic disk in the solar neighborhood, leading to possible conclusions on the nature of the Dark Matter in our Galaxy. Astrophysical applications also include, among many others, detection of extra-solar planetary systems and tests of general relativity.

The Global Astrometric Interferometer for Astrophysics (GAIA) (Lindegren et al. 1994; Lindegren \& Perryman 1995) is a space-based instrument dedicated to global astrometry, following on the success of Hipparcos (Perryman et al. 1992) and aiming at the enhancement by a factor 100, compared to Hipparcos, not only for the astrometric precision but also for the number of objects observed. GAIA's main objective is to perform global astrometry at the 5-20 $\mu$ as level for positions, proper motions and parallaxes on $\sim 50$ million objects. It could comprise three interferometers, stacked on top of each other and set at large angles to each other, so as to bring together regions of the sky separated by long arcs. Like Hipparcos, GAIA would be a continuously rotating instrument for which the calibration parameters of the in- strument are part of the astrometric data, solved for during the global data reduction. Unlike Hipparcos, each of GAIA's interferometers will observe several stars simultaneously, recording their positions relative to a focal grid by means of pupil plane detectors.

The downside of this choice of continuous scanning is the intrinsic limitation of the integration time on any observed object: its upper bound is roughly $(\Omega T) / 4 \pi$ where $\Omega$ is the solid angle subtended by the field-of-view of the detector and $T$ the total duration of the mission. Since the latter is not expandable, the field-of-view of the instrument (of each of the three identical interferometers) should be maximized. Simple calculations on astrometric precision for photon noise limited observations show that a field-of-view of nearly 1 degree in diameter is needed to open the possibility of reaching the desired astrometric precision. The Fizeau type interferometer traditionally offers a much wider field-of-view than the Michelson type, hence our choice of optical configuration. In the following sections, a design for a three-mirror Fizeau interferometer, diffraction limited over a field-of-view of 0.9 degree is described.

\section{Modelling of the instrument}

\subsection{An all-reflective compact three-mirror telescope}

The natural first step to the design of a Fizeau interferometer is the design of the corresponding mono-pupil telescope on which a two-aperture mask will later be placed. The starting point of the design was set by the need for a wide field of view and by its necessary compactness: it had to comply with the dimensions of the Ariane $\mathrm{V}$ payload shroud which is a $4.5 \mathrm{~m}$ circular envelope. For these reasons, it seemed logical to consider a three-mirror telescope, capable of being very compact with a sufficiently long focal length. A reasonable size of a few decimeters for the focal plane sets the value of the latter: with a field-of-view of $\sim 1^{\circ}$, a value between 10 and $15 \mathrm{~m}$ seems adequate.

Adding a third mirror to the system introduces additional degrees of freedom compared to a two-mirror design, making it possible to obtain an aplanatic and anastigmatic configuration (Korsh 1977). Solutions for threemirror designs corrected for spherical aberration, coma, astigmatism and field curvature have appeared in the literature (Wetherell \& Rimmer 1972; Korsh 1972, 1977; Gelles 1973; Eisenberg \& Pearson 1987). Three-mirror designs optimized with automatic ray-trace programs have also been proposed (Robb 1978) but their dimensions exceed our limitations.

So far, none of the proposed designs meets our needs. For instance, Korsh (1977) proposed an anastigmatic design with an overall length of $4.5 \mathrm{~m}$ for a clear aperture of $1.5 \mathrm{~m}$. A two-mirror, three-surface telescope, very similar to our design, is given by Eisenberg \& Pearson (1987) but has an overall length of $4.32 \mathrm{~m}$, a field-of-view of 


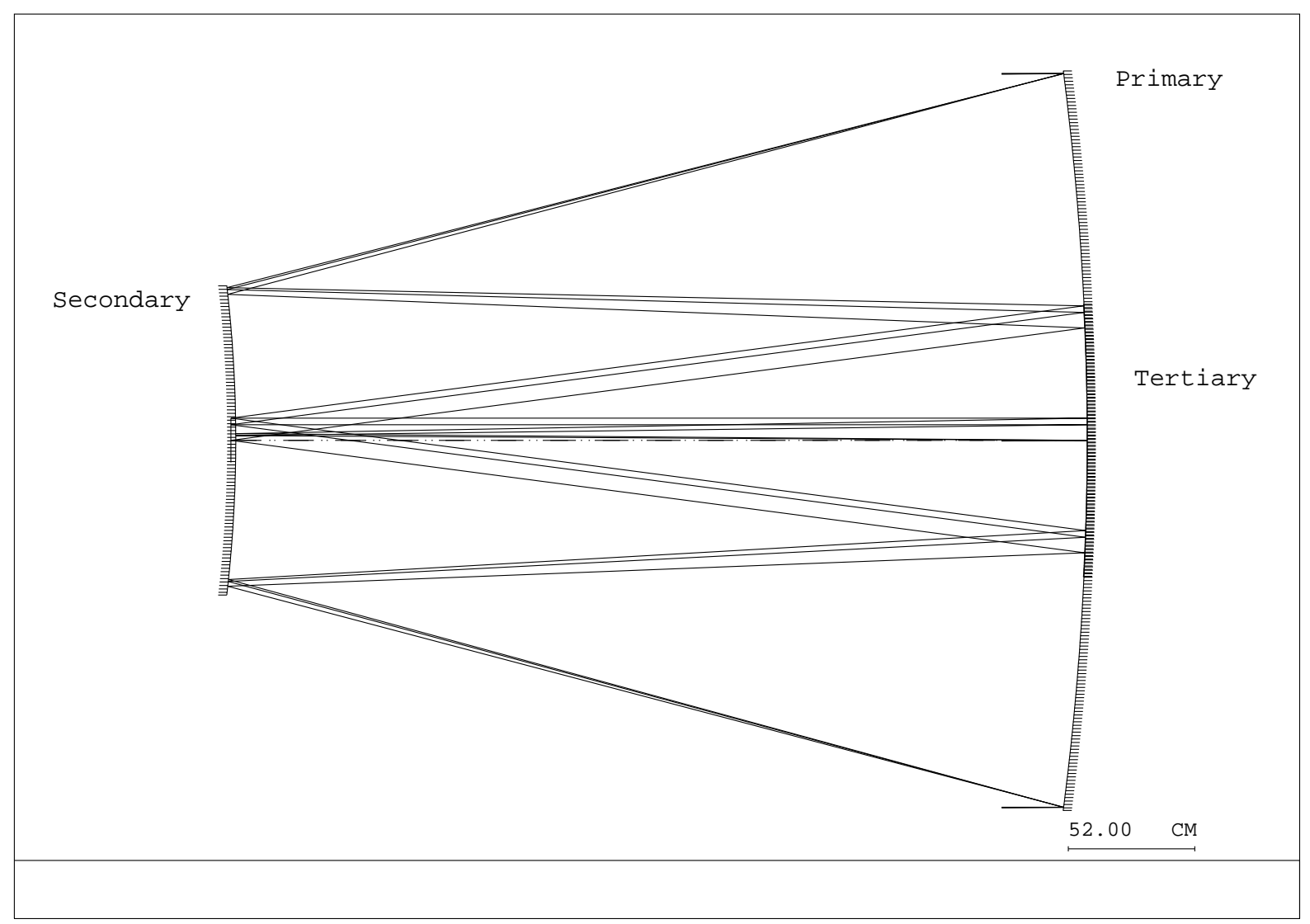

Fig. 1. Cross section of the three-mirror design. The primary and the tertiary mirror share the same vertex. The overall length of the telescope is $3.5 \mathrm{~m}$ for a primary diameter of $3 \mathrm{~m}$. The final focal ratio is 3.85

10 arcmin and no detailed study of system aberrations is given. The design we propose is not new in its aspect (Rumsey 1969a, b; Eisenberg \& Pearson 1987) but we believe that such a compact instrument, diffraction-limited over a $0.9^{\circ}$ field-of-view has not before been presented.

Our design constraints were to use 3 conic mirrors, to achieve a diffraction-limited field-of-view of $0.9^{\circ}$, maintain a total length no greater than $3.5 \mathrm{~m}$, and to constrain the effective focal length to $11.55 \mathrm{~m}$. We optimized for the best composite focus over the whole $0.9^{\circ}$ field-of-view, letting the conic constants, separations, and focal lengths of the mirrors vary.

In the final configuration, the three mirrors composing the telescope are hyperboloids with reasonable conic constants. The specifications of the mirrors are shown in Table 1 . The tertiary and the primary share a common vertex but have different curvatures. The aperture stop is located on the primary mirror. The focal plane is located $2 \mathrm{~cm}$ behind the secondary. Its proximity to the instrument's envelope could facilitate thermal control, passive or active. The overall length of the telescope is $3.5 \mathrm{~m}$ and its width is $3 \mathrm{~m}$, thus fitting in the Ariane $\mathrm{V}$ envelope. Us-
Table 1. Main parameters of the three-mirror telescope from which the Fizeau interferometer is derived. $z=$ coordinate of the vertex of the surface, $D=$ outer diameter of surface, $r=$ radius of curvature, $K=$ conic constant. Light enters in the $+z$ direction. All mirrors are concave towards $+z$ (negative curvature). The focal surface is flat

\begin{tabular}{lccrc}
\hline Surface & $z$ & $D[\mathrm{~m}]$ & \multicolumn{1}{c}{$r$} & $K$ \\
\hline primary mirror & 3.51996519 & 3.00 & 11.57 & -1.407347 \\
secondary mirror & 0.01996519 & 1.25 & 5.36 & -3.581175 \\
tertiary mirror & 3.51996519 & 1.12 & 10.02 & -5.086847 \\
focal plane & 0.00 & 0.18 & $\infty$ & - \\
\hline
\end{tabular}

ing the longest possible separations between the mirrors inside the telescope structure makes it possible to have relatively slow beams, better for aberrations. The final focal ratio of the system is 3.85 . The optical layout of the telescope is shown in Fig. 1. 
At the typical effective wavelength, $550 \mathrm{~nm}$, the diameter of the first Airy ring of the diffraction pattern of the telescope is $5.17 \mu \mathrm{m}$. The rms sizes of the geometrical spots given by Code $\mathrm{V}^{1}$ are $2.5 \mu \mathrm{m}$ on axis, $4.3 \mu \mathrm{m}$ at 0.32 degree (corresponding to $0.45 / \sqrt{2}$ ) and $5.5 \mu \mathrm{m}$ at 0.45 degree, making it almost diffraction limited over a FOV of 0.9 degree. The Strehl ratio (ignoring the central obscuration) at full field is 0.875 . The spot diagram is displayed in Fig. 2.

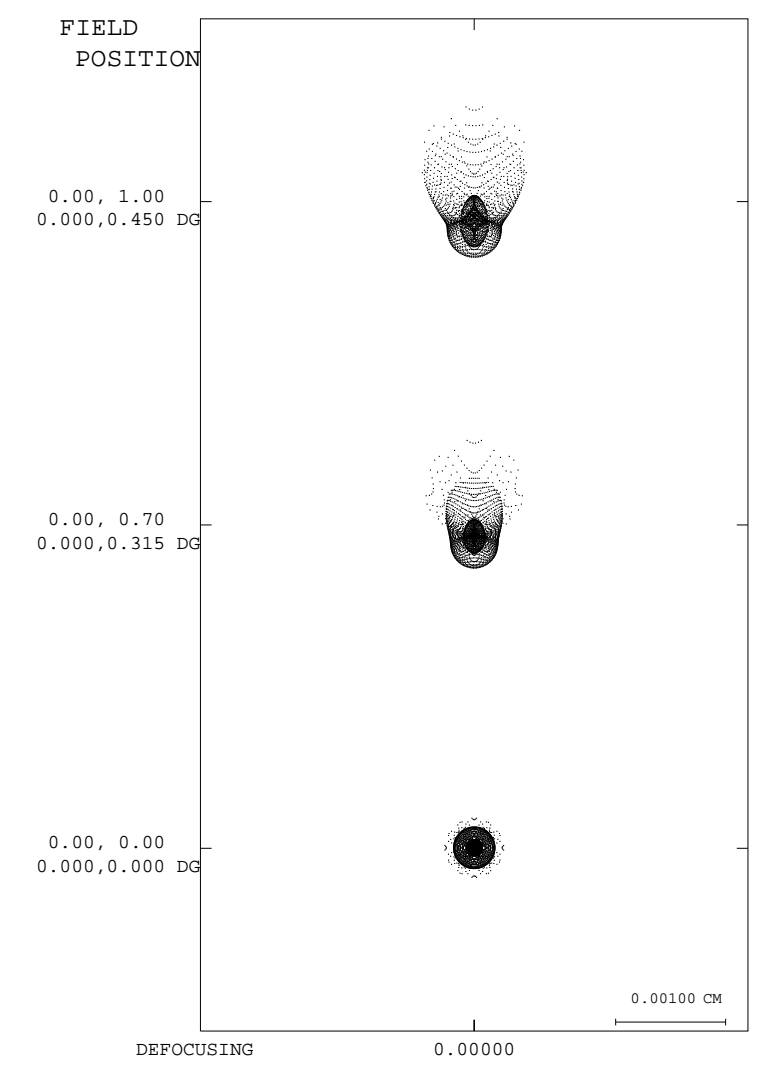

Fig. 2. Geometrical spot diagram for the full-aperture telescope. The rms spot sizes are $2.5,4.3$ and $5.5 \mu \mathrm{m}$ for the three field angle $0,0.32$ and 0.45 degree

Even at full field, these aberrations have an amplitude of $\sim 5 \mu \mathrm{m}$, less than the Airy disk diameter of the telescope. Although the nominal instrument has very small aberrations, a precise optical modelling of the system is required to allow the prediction of the aberrations, and in particular the distortion as discussed in Sect. 3.4, as a function of the perturbations applied to the system. It should be noted that the aberrations of the corresponding Fizeau interferometer, as described in the following section, are often significantly smaller than those of the corresponding full-aperture system.

\footnotetext{
${ }^{1}$ Code $\mathrm{V}$ is a registered product of Optical Research Associates, Pasadena, California.
}

\subsection{Formalism for fringe and visibility calculation}

The ray sampling densities of most optical design programs are inadequate to characterize the performance of a Fizeau interferometer. We desire a large number of rays across two widely separated and relatively small subapertures, while at the same time demanding diffraction analysis using both sub-apertures simultaneously for interferometry. Thus we turned to another tool to transform our conventional telescope into an interferometer. The Controlled Optics Modelling Package (COMP; Redding et al. 1992) offers a much larger number of rays (up to $2048 \times 2048$ ) and the possibility of implementing small sub-apertures by means of obscurations on our primary mirror. Moreover, it is possible to have full diffraction propagation with COMP, making it possible to obtain diffraction patterns and in particular interference fringes.

A layout of the Fizeau interferometer as we designed it is shown in Fig. 3. Each of the three original mirrors could be reduced to two small sub-apertures. For the primary, these apertures have a diameter of $40 \mathrm{~cm}$. For the two other optical surfaces, they are a bit smaller $(\sim 25 \mathrm{~cm}$ in diameter).

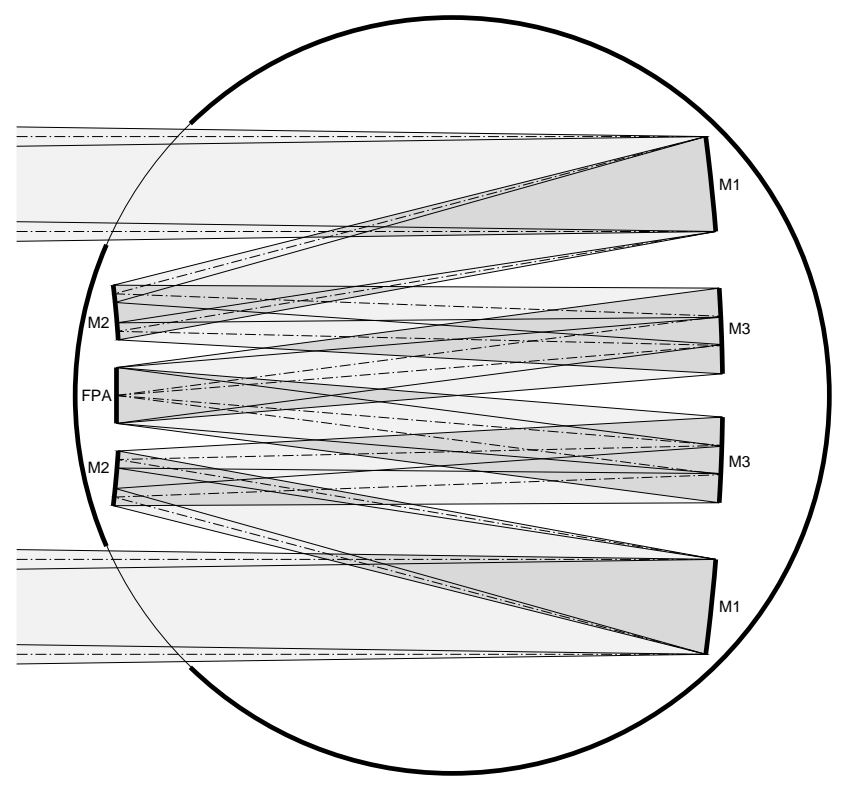

Fig. 3. Optical layout of the Fizeau interferometer. The $4.5 \mathrm{~m}$ envelope of the Ariane $\mathrm{V}$ launcher is shown as well

The purpose of building an interferometer is to measure the phase and amplitude of the interference fringes resulting when a star is observed. The phase gives an extremely sensitive measure of the star's position relative to other stars (whose phases are being simultaneously measured), while the modulation amplitude is related to the signal-to-noise ratio of the measurement. In an ideal instrument, the two sub-apertures form perfectly overlapping, unaberrated Airy patterns, and the amplitude of the 
fringes resulting from their superposition is maximized. In the real case, however, the large field-of-view and limited degrees of freedom have led to a design that introduces some aberrations and image separation. Perturbations such as sub-aperture tilts, displacements, and defocus will further reduce the fringe modulation. In this section, we present the formalism for analyzing the fringe modulation. The next section then utilizes these results and presents a tolerance analysis of the design.

We begin by assuming that the incoming light is monochromatic and that it emanates from a distant point source. The assumption of an unresolved source is reasonable since the vast majority of stars observed by GAIA are unresolved with a $3 \mathrm{~m}$ baseline.

The aberrations of the instrument are conventionally represented by the equivalent phase shifts $\phi_{1}$ and $\phi_{2}$ in the two sub-apertures. The complex amplitude of the incoming plane wavefront is then described by the two functions

$$
P_{n}(\boldsymbol{r})=a \mathrm{e}^{-i k \cdot r} P\left(\boldsymbol{r}+(-1)^{n} \boldsymbol{B} / 2\right) \mathrm{e}^{-i \phi_{n}(r)}
$$

$(n=1,2)$ where:

$-a$ is a constant of the incident field strength

$-\boldsymbol{r}$ is the coordinate in the pupil plane which has its origin at the center of the line joining the apertures

$-\boldsymbol{k}=(2 \pi / \lambda) \boldsymbol{u}$ is the wave vector (i.e. the wavefront is moving in direction $\boldsymbol{u}$ when it is incident on the subapertures)

$-P$ is the sub-aperture pupil i.e. $P=1$ for $r=|\boldsymbol{r}|<d / 2$ ( $d$ being the sub-aperture diameter), $P=0$ if not

$-\boldsymbol{B}$ is the baseline vector joining the centers of the two pupils

The optical system performs a Fourier Transform of the wavefront, resulting in an image plane electric field given by $\tilde{P}_{1}+\tilde{P}_{2}$, where tilde over a symbol indicates the Fourier Transform of its corresponding function in the pupil plane. The Fourier Transform of $P_{n}(\boldsymbol{r})$ is defined as

$$
\tilde{P}_{n}(\boldsymbol{s})=\int P_{n}(\boldsymbol{r}) \exp [-i(2 \pi / \lambda) \boldsymbol{r} \cdot \boldsymbol{s}] \mathrm{d} \boldsymbol{r}
$$

where $s$ represents the angular coordinates in the image plane.

The observed intensity is proportional to the square of the field, so that one has:

$$
I=\left|\tilde{P}_{1}\right|^{2}+\left|\tilde{P}_{2}\right|^{2}+2 \operatorname{Re}\left(\tilde{P}_{1} \tilde{P}_{2}^{*}\right)
$$

The signal modulation is proportional to the amplitude of the cross term.

It is convenient to analyze the fringes by taking the Fourier Transform of their intensity distribution. Squaring it, we get the spectral density (or power spectrum) of the image, which presents non-overlapping low and high frequency terms. Roddier \& Léna (1984) have shown that the ratio of high to low frequency energies gives a good estimation of $\left(V^{2} / 2\right)$. Their equations include the effects of resolving the source with the interferometer baseline but with a perfect system with no aberrations. We show below, in Sect. 3.3, that this ratio is proportional to a ratio of convolution integrals of intensities displaced by perturbations applied to the system. We applied this method to estimate the visibility of the fringes given by COMP. An image obtained with COMP on the focal plane can be seen in Fig. 4. The corresponding intensity curve is

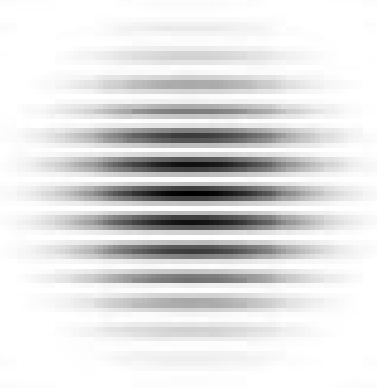

Fig. 4. Interference fringes obtained with COMP displayed in inverted colors. The fringe spacing at $0.55 \mu \mathrm{m}$ for an interferometer with $40 \mathrm{~cm}$ apertures and $2.6 \mathrm{~m}$ baseline is 43.6 mas. There are 16 fringes within the central peak of the Airy pattern which has an angular width of 691.8 mas. The central peak and first bright ring only are shown

displayed in Fig. 5. The method then consisted of taking the 2-D Fourier Transform of the fringes, squaring its modulus and calculating the ratio of appropriate terms. This spectral density is displayed in Fig. 6. The fringe contrast we derived is 0.995 on axis, 0.989 at $0.32^{\circ}$ and 0.984 at $0.45^{\circ}$ where angles are in the direction of the baseline axis, $Y$. The contrasts corresponding to angles in the perpendicular direction $(X)$ are respectively 0.995 , $0.986,0.974$. These values correspond to monochromatic fringes $(\lambda=0.55 \mu \mathrm{m})$.

\subsection{The aberration-free case}

We first consider the ideal case where there are no aberrations so that $\phi_{n}=0$. One then has

$$
\tilde{P}_{1}=\tilde{P}(\rho) \mathrm{e}^{-i \pi \rho \cdot B / \lambda}
$$




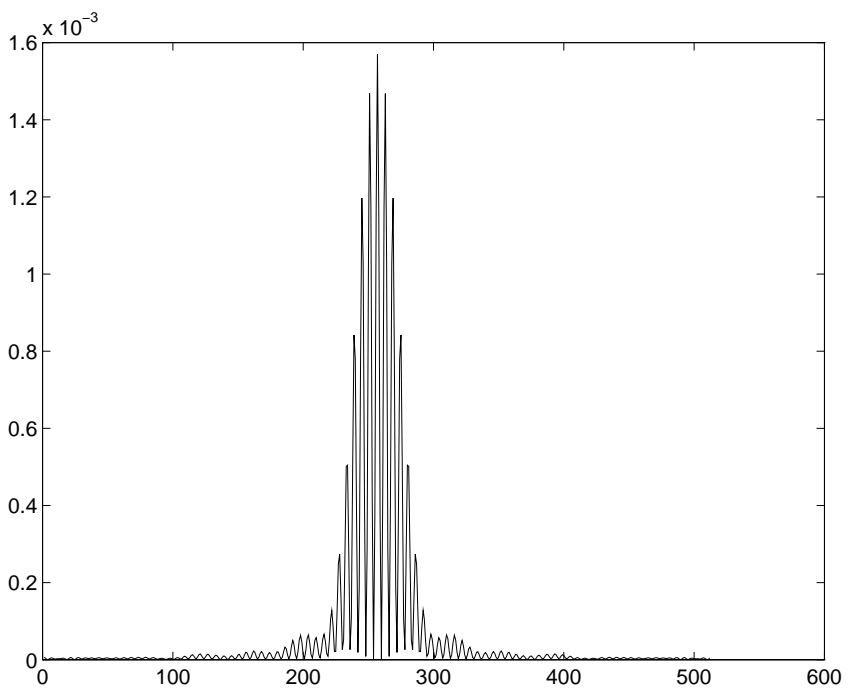

Fig. 5. Fringes integrated along the direction perpendicular to the direction of the baseline, for on-axis observation. The $x$-axis scale is arbitrary

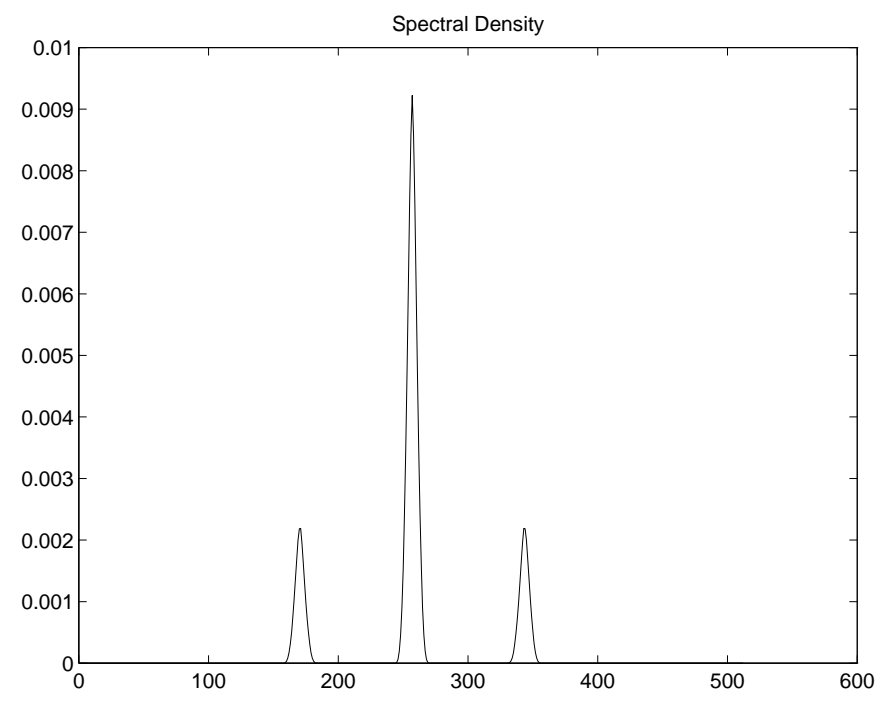

Fig. 6. Spectral density of the image

$$
\tilde{P}_{2}=\tilde{P}(\rho) \mathrm{e}^{i \pi \rho \cdot B / \lambda}
$$

and

$$
\tilde{P}(\rho)=a \frac{2 J_{1}(\pi \rho d / \lambda)}{\pi \rho d / \lambda}
$$

where:

$-\boldsymbol{\rho}=\boldsymbol{s}-\boldsymbol{u}$ and $\rho=|\boldsymbol{\rho}|$

- $J_{1}$ is a first-order Bessel function

The intensity on the focal plane is then given by

$$
I=\left(a \pi \frac{d^{2}}{4}\right)^{2}\left[\frac{2 J_{1}(\pi \rho d / \lambda)}{\pi \rho d / \lambda}\right]^{2}[1+\cos ((2 \pi / \lambda) \boldsymbol{\rho} \cdot \boldsymbol{B})]
$$

and we obtain the classical result of interference fringes corresponding to a baseline $B$ modulated by an Airy pattern corresponding to an aperture $d$.

\subsection{A simplified model for the aberrated system}

In the case where the only aberration is a tilt across each sub-aperture (as would happen given a small mechanical perturbation of the optical elements), it is convenient to model the aberrations of the pupil by

$$
\phi_{n}(\boldsymbol{r})=(2 \pi / \lambda) \boldsymbol{\alpha}_{n} \cdot \boldsymbol{r}
$$

The Fourier Transform of each sub-aperture is then

$$
\begin{aligned}
& \tilde{P}_{1}=\tilde{P}\left(\left|\boldsymbol{\rho}-\boldsymbol{\alpha}_{1}\right|\right) \mathrm{e}^{-i \pi\left(\rho-\alpha_{1}\right) \cdot B / \lambda} \\
& \tilde{P}_{2}=\tilde{P}\left(\left|\boldsymbol{\rho}-\boldsymbol{\alpha}_{2}\right|\right) \mathrm{e}^{i \pi\left(\rho-\alpha_{2}\right) \cdot B / \lambda}
\end{aligned}
$$

and the cross term which appears in the calculation of the resulting intensity is

$$
2 \operatorname{Re}\left(\tilde{P}\left(\left|\boldsymbol{\rho}-\boldsymbol{\alpha}_{1}\right|\right) \tilde{P}^{*}\left(\left|\boldsymbol{\rho}-\boldsymbol{\alpha}_{2}\right|\right) \mathrm{e}^{-2 i \pi\left(\rho-\left(\alpha_{1}+\alpha_{2}\right) / 2\right) \cdot B / \lambda}\right)
$$

The expression of the intensity on the focal plane is then

$$
\begin{aligned}
I(\boldsymbol{\rho})= & \left(\left|\tilde{P}\left(\left|\boldsymbol{\rho}-\boldsymbol{\alpha}_{1}\right|\right)\right|^{2}+\left|\tilde{P}\left(\left|\boldsymbol{\rho}-\boldsymbol{\alpha}_{2}\right|\right)\right|^{2}\right) \\
& \times\left[1+\frac{2\left|\tilde{P}\left(\left|\boldsymbol{\rho}-\boldsymbol{\alpha}_{1}\right|\right) \tilde{P}^{*}\left(\left|\boldsymbol{\rho}-\boldsymbol{\alpha}_{2}\right|\right)\right|}{\left|\tilde{P}\left(\left|\boldsymbol{\rho}-\boldsymbol{\alpha}_{1}\right|\right)\right|^{2}+\left|\tilde{P}\left(\left|\boldsymbol{\rho}-\boldsymbol{\alpha}_{2}\right|\right)\right|^{2}}\right. \\
& \left.\times \cos \left(2 \pi\left(\boldsymbol{\rho}-\left(\boldsymbol{\alpha}_{1}+\boldsymbol{\alpha}_{2}\right) / 2\right) \cdot \boldsymbol{B} / \lambda\right)\right]
\end{aligned}
$$

which can be written in the form

$$
\begin{aligned}
I\left(\boldsymbol{\rho}, \boldsymbol{\alpha}_{1}, \boldsymbol{\alpha}_{2}\right) & =I_{0}\left(\boldsymbol{\rho}, \boldsymbol{\alpha}_{1}, \boldsymbol{\alpha}_{2}\right) \\
& \times\left(1+V\left(\boldsymbol{\rho}, \boldsymbol{\alpha}_{1}, \boldsymbol{\alpha}_{2}\right) \cos \left(2 \pi \boldsymbol{f}_{\mathrm{o}} \cdot \boldsymbol{\rho}-\psi\right)\right)
\end{aligned}
$$

where $\boldsymbol{f}_{\mathrm{o}}=\boldsymbol{B} / \lambda$ is the (vectorial) spatial frequency corresponding to the baseline $\boldsymbol{B}, \psi=(\pi / \lambda)\left(\boldsymbol{\alpha}_{1}+\boldsymbol{\alpha}_{2}\right) \cdot \boldsymbol{B}$ and $V\left(\boldsymbol{\rho}, \boldsymbol{\alpha}_{1}, \boldsymbol{\alpha}_{2}\right)$ account for the aberrations and perturbations of the system. This shows that there is a loss of visibility due to the nature of the instrument. This contrast term reflects the optical transfer function of the interferometer itself. The piston term that would have to be introduced in a more precise model of the aberrations would create a phase shift of the fringes. Piston leads to distortion and we handle that issue in Sect. 3.4.

\subsection{The effect of finite bandwidth}

With a finite optical bandwidth $\Delta \lambda$, the loss of visibility is slightly increased. Indeed, assuming equal power at all frequencies, the reduction factor due to chromatic effects that should be applied to the theoretical contrast can be expressed as:

$$
\Gamma_{\Delta \lambda}=\left|\frac{\sin \left(\pi \frac{\Delta l \Delta \lambda}{\lambda_{0}^{2}}\right)}{\pi \frac{\Delta l \Delta \lambda}{\lambda_{0}^{2}}}\right|
$$


where $\Delta l$ is the optical path difference between the two interfering beams. If a bandwidth equal to $\Delta \lambda=\lambda / 10$ is assumed, an OPD of the same value would lead to a negligible reduction of the fringe contrast, but an OPD of $\lambda$ (still a tenth of the coherence length) gives a correction factor of 0.986 . For a bandwidth $\Delta \lambda=\lambda / 4$, the coherence length is $4 \lambda$ and an OPD of $\lambda$ reduces the fringe visibility by a factor 0.900 . The impact of polychromaticity becomes more important at large field angles.

Our program makes it possible to co-add interference patterns obtained for different wavelengths. Table 2 gives the fringe contrast obtained with bandwidths of 60 and 100 nanometers, for the unperturbed system, at three field angles. As predicted, depending on the field angle, the reduction factor on the fringe visibility ranges from 0.8 to 0.9 for a bandwidth equal to $\Delta \lambda \sim \lambda / 9(60 \mathrm{~nm})$, even at the edge of the field for a slightly perturbed system. For a wider bandwidth, $\Delta \lambda \sim \lambda / 5(100 \mathrm{~nm})$, the fringe contrast is reduced by the same order of magnitude for the unperturbed system. After perturbation of the system, the consequences of such a bandwidth are, of course, more dramatic.

Table 2. Variations of the fringe contrast with field angles for polychromatic fringes with two different optical bandwidths, for an unperturbed system

\begin{tabular}{lccc}
\hline Field angle [deg] & 0 & 0.32 & 0.45 \\
\hline$\Delta \lambda \sim \lambda / 5$ & 0.822 & 0.764 & 0.781 \\
$\Delta \lambda \sim \lambda / 9$ & 0.887 & 0.812 & 0.823 \\
\hline
\end{tabular}

Table 3. Variations of the fringe contrast with field angles in a plane containing the optical axis and the baseline of the interferometer $(Y-Z$ plane) and in a perpendicular plane $(X-Z$ plane), for the unperturbed system

\begin{tabular}{lcccc}
\hline \multirow{2}{*}{ Field angles } & $0^{\circ}$ & $0.32^{\circ}$ & $0.45^{\circ}$ & $0.8^{\circ}$ \\
\hline$Y-Z$ plane & 0.995 & 0.989 & 0.984 & 0.980 \\
$X-Z$ plane & 0.995 & 0.986 & 0.974 & 0.888 \\
\hline
\end{tabular}

\section{Tolerances of the design}

It is of prime importance, when studying the feasibility of an optical instrument, to define the margin of freedom for variations from the nominal position of each element of the system. In this section, we monitor the variations of the fringe visibility when each optic is mechanically perturbed in all directions and independently from the others. These perturbations could correspond to thermal variations from one end of the interferometer to the other. It is then possible to choose a quality criterion for the fringe visibility which sets the tolerances on the structure of the instrument.

\subsection{Theoretical structure and thermal variations}

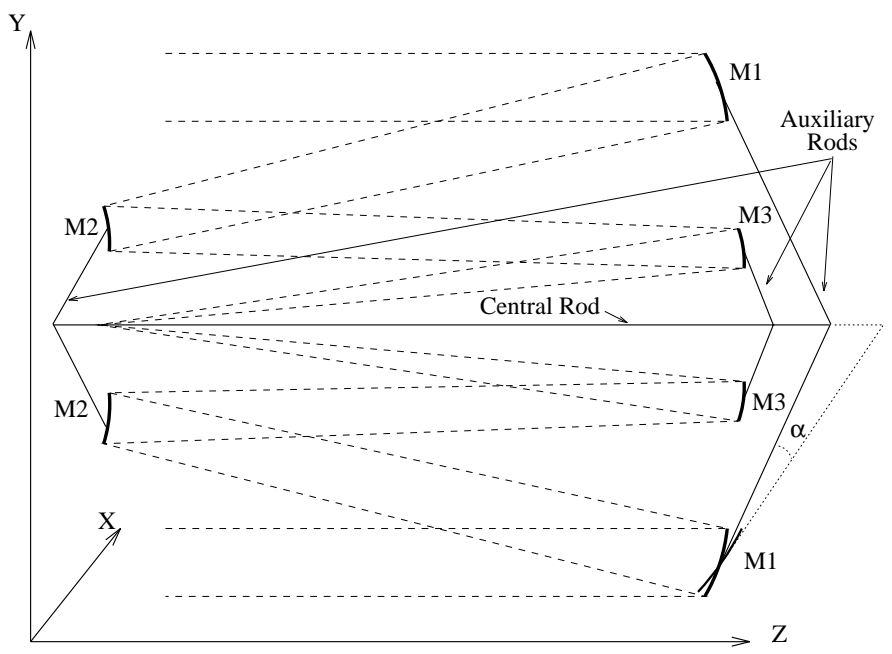

Fig. 7. Theoretical structure made of very low expansion material and supporting the optical system used as a rule-of-thumb for the derivation of the tolerances. It is composed of a central rod and several secondary rods supporting the sub-apertures on the three surfaces. An exaggerated expansion of the central rod provoking a rotation $\alpha$ of the lower auxiliary rod, supporting a primary sub-aperture, is drawn as well, showing the kind of perturbation we imagined to derive the tolerances of the instrument

We tried to derive some kind of "rule of thumb" in order to estimate the expected displacements due to thermal variations. To do so, we considered a structure made of very low expansion material, supporting the different elements of the interferometer. The way we imagined it is displayed in Fig. 7. This schematic structure comprises a number of rods, each connected to a central rod. Each optical element is free to rotate around its vertex or to be translated. To get the order-of-magnitude of the tilts these optics would suffer from, we considered translations of the central rod (or an expansion of it due to thermal variations) that would rotate the off-axis mirrors around their vertices without translating them, as shown in Fig. 7 where $\alpha$ is the resulting rotation due to the translation of the central rod.

To estimate the values of these translations and rotations, we considered a Coefficient of Thermal Expansion (CTE) of $10^{-8} \mathrm{~K}^{-1}$, which seems quite reasonable today. The spatial homogeneity of the CTE and its variations with the temperature are not considered here. A temperature variation of $30 \mathrm{~K}$ for instance (which is purposely chosen as extremely large), will expand the $3.5 \mathrm{~m}$ central 
rod by $10^{-8} \times 30 \times 3.5=1.05 \mu \mathrm{m}$. The resulting rotation for the primary sub-apertures would be $1.05 / 1.3=0.8$ microradian $(\mu \mathrm{rad})$. This value is rather pessimistic considering the performances of active thermal control. It could easily be divided by a very significant factor (probably more than 100), depending also on the value of the CTE whose range is $5.10^{-9}-5 \cdot 10^{-8} \mathrm{~K}^{-1}$ for very low expansion material at the considered temperatures. A value of $10 \mathrm{~K}$ for temperature variation can give us a first idea of the tolerances. This brings the previous value to $0.35 \mu \mathrm{m}$ for the central rod and to $0.27 \mu \mathrm{rad}$ for the tip or tilt of a primary aperture.

\subsection{Perturbations and resulting contrasts}

In the case of a multi-pupil instrument, such as an interferometer, it is essential to look at perturbations of individual optical elements of the system as opposed to a global tip/tilt or translation of the whole telescope. Driving COMP and SCOMP (Subroutine COMP) by a fortran program, we propagated rays and diffraction through both apertures separately, making it possible to perturb each optical element independently. The amplitudes of the propagated electric fields are then added and squared to obtain the diffraction pattern on the common focal plane. The fringes are treated and analyzed as exposed previously. The term "perturbations" includes tips, tilts and translations in various directions. As shown in Fig. 7, the optical axis is the $Z$ axis and the axis supporting the baseline of the interferometer is $Y$. A $Y$-tilt means that the element rotates around the axis parallel to the $X$ axis and passing through its vertex. Field angles are usually in the $Y-Z$ plane except when mentioned otherwise. The fringe contrast for various field angles is summarized in Table 3.

The contrast of these monochromatic fringes stays high at all field angles in both viewing direction, even up to 0.8 degree in the field (contrast $=0.98$ and 0.88 ), confirming the good performances of the design.

Each of the three mirrors are split into two smaller subapertures. The amplitude of the perturbations is derived using our theoretical structure as a "rule-of-thumb". As mentioned before, an expansion of $0.35 \mu \mathrm{m}$ could lead to a tilt of $0.27 \mu \mathrm{rad}$ for a primary sub-aperture. The same expansion would tilt a secondary sub-aperture by $0.65 \mu \mathrm{rad}$ and a tertiary one by $0.875 \mu \mathrm{rad}$. The fringe visibilities obtained after perturbation of the system are summarized in Table 4 . Concerning primary apertures perturbations, the slightly different values for the visibility after positive and negative $Y$-tilts are due to the fact that initially, the centroids of the images formed by each aperture do not perfectly overlap, inducing a non-symmetrical situation. A negative tilt improves the initial configuration and thus enhances the fringe contrast. The latter remains greater than 0.88 for perturbations of amplitude up to $0.3 \mu \mathrm{rad}$, which is rather satisfying.
As for off-axis observations after perturbation of a primary sub-aperture, a $0.3 \mu \mathrm{rad}$ tilt leads to a contrast of 0.85 at 0.32 degree in the field. Since the system has a wide diffraction limited field-of-view, one can expect the contrast to be quite constant over the whole field after any kind of perturbations: the variation is less than $5 \%$ of the on-axis value of the visibility. It is interesting to apply the same kind of perturbations to the secondary and tertiary sub-apertures. Visibility values after perturbation of these elements are also given in Table 4 where it can be seen that tolerances on secondary and tertiary elements seem less stringent than on the primary ones: the loss of visibility after various perturbations ( $X$-tilt, $Y$-tilt, $Z$-shift) of amplitude corresponding to the thermal expansion we considered, remain smaller than $10 \%$. For nominal values corresponding to a $10 \mathrm{~K}$ temperature variation, the contrast stays above 0.83 for a single perturbation. A perturbation applied to two elements of the system has much more dramatic consequences on the fringe visibility. For instance, tilting a primary and a tertiary aperture by the nominal values given above $(0.3$ and $0.875 \mu \mathrm{rad}$ respectively) leads to a contrast of 0.66 , probably insufficient in terms of signal-to-noise ratio.

\subsection{A relationship between fringe visibility and centroid separation}

In an optical system such as a Fizeau interferometer, the contrast of the fringes obtained in the focal plane depends on two main parameters: the optical aberrations of the system and the degree of super-imposition of the images formed by each aperture. As a matter of fact, each of the two apertures of the interferometer produces a diffraction pattern - an Airy spot convolved with a transfer function - on the focal plane. Interference fringes are the result of the coherent super-imposition of these two images. For the unperturbed system, Fig. 8 shows the geometrical spot diagram of the interferometer: the centroids of the two spots are separated by $\sim 2 \mu \mathrm{m}$, i.e. about a twentieth of the Airy disk diameter.

The quality of the fringes depends directly on how well the images overlap. This can be quantified by the measure of the linear separation between the centroids of the spots given by each aperture. In a system with low aberrations, or in a low-aberration regime, the visibility of the fringes is uniquely function of this separation. To prove empirically this relationship, we plot the fringe visibility as a function of the separation of the centroids, which positions are calculated for each configuration of the optical elements, for each family of perturbations. The curves show an excellent agreement. Moreover, it could be fitted using another way to express the contrast $V$ as a function of spot centroid separation. 
Table 4. Fringe visibilities for various perturbations applied independently to the sub-apertures of all three mirrors. In certain cases, visibilities are given for several field angles. $Y$-shift and $Z$-shift represent translations along $Y$ and $Z$ axes. Tilts are described in the text

\begin{tabular}{|c|c|c|c|c|c|c|c|c|c|c|}
\hline \multirow[t]{2}{*}{ Mirror } & \multirow{2}{*}{$\begin{array}{l}Y \text {-tilt } \\
{[\mu \mathrm{rad}]}\end{array}$} & \multirow{2}{*}{$\begin{array}{c}Y \text {-shift } \\
{[\mu \mathrm{m}]}\end{array}$} & \multicolumn{4}{|c|}{ Visibility } & \multirow{2}{*}{$\begin{array}{l}X \text {-tilt } \\
{[\mu \mathrm{rad}]}\end{array}$} & \multirow{2}{*}{$\begin{array}{l}\text { Visibility } \\
\text { on- axis }\end{array}$} & \multirow{2}{*}{$\begin{array}{c}Z \text {-shift } \\
{[\mu \mathrm{m}]}\end{array}$} & \multirow{2}{*}{$\begin{array}{c}\text { Visibility } \\
\text { on-axis }\end{array}$} \\
\hline & & & on-axis & $0.32^{\circ}$ & $0.45^{\circ}$ & $0.8^{\circ}$ & & & & \\
\hline \multirow[t]{11}{*}{ primary } & 0.1 & & 0.975 & & & & 0.1 & 0.987 & -1.0 & 0.992 \\
\hline & 0.3 & & 0.887 & 0.863 & 0.845 & 0.839 & 0.3 & 0.922 & -0.5 & 0.994 \\
\hline & 0.6 & & 0.633 & & & & 0.6 & 0.689 & 0.5 & 0.996 \\
\hline & 0.7 & & 0.532 & & & & 0.7 & 0.589 & 1.0 & 0.997 \\
\hline & 0.8 & & 0.441 & & & & 0.8 & 0.482 & 1.5 & 0.998 \\
\hline & -0.1 & & 0.999 & & & & & & & \\
\hline & -0.3 & & 0.965 & & & & & & & \\
\hline & -0.6 & & 0.790 & & & & & & & \\
\hline & -0.7 & & 0.698 & & & & & & & \\
\hline & -0.8 & & 0.597 & & & & & & & \\
\hline & & 1.0 & 0.979 & & & & & & & \\
\hline \multirow{2}{*}{ secondary } & 0.65 & & 0.907 & 0.884 & 0.868 & & 0.65 & 0.888 & 1.0 & 0.926 \\
\hline & & 1.0 & 0.937 & & & & & & & \\
\hline \multirow[t]{7}{*}{ tertiary } & 0.1 & & 0.990 & & & & & & 1.0 & 0.927 \\
\hline & 0.3 & & 0.976 & & & & 0.3 & 0.927 & & \\
\hline & 0.6 & & 0.944 & & & & 0.6 & 0.910 & & \\
\hline & 0.8 & & 0.914 & & & & & & & \\
\hline & 0.875 & & 0.900 & 0.878 & 0.862 & & & & & \\
\hline & 1.0 & & 0.876 & 0.851 & 0.834 & & & & & \\
\hline & & 1.0 & 0.929 & & & & & & & \\
\hline
\end{tabular}

Let us consider again the Fourier Transform (denoted here by $F$ for clarity) of the intensity $I$ :

$$
\begin{aligned}
\tilde{I} & =F\left(\left|\tilde{P}_{1}\right|^{2}\right)+F\left(\left|\tilde{P}_{2}\right|^{2}\right)+F\left(\tilde{P}_{1} \tilde{P}_{2}{ }^{*}+\tilde{P}_{1}{ }^{*} \tilde{P}_{2}\right) \\
& =P_{1} \star P_{1}^{*}+P_{2} \star P_{2}^{*}+P_{1} \star P_{2}^{*}+P_{1}^{*} \star P_{2}
\end{aligned}
$$

where $\star$ denotes the convolution of two distributions. This equation contains a low frequency term (the sum of the auto-correlations) and high-frequency terms centered at $\pm \boldsymbol{B}$. The terms do not overlap, enabling us, by squaring this expression, to obtain:

$$
|\tilde{I}|^{2}=\left|P_{1} \star P_{1}^{*}+P_{2} \star P_{2}^{*}\right|^{2}+\left|P_{1} \star P_{2}^{*}\right|^{2}+\left|P_{1}^{*} \star P_{2}\right|^{2}
$$

We mentioned previously that Roddier \& Léna (1984) had shown that the ratio of the high to low frequency energies gave a good estimation of $V^{2} / 2$. Thus we define the modulation $C$ of the fringes:

$$
C=\frac{V^{2}}{2}=\frac{\iint\left|P_{1} \star P_{2}^{*}\right|^{2}+\left|P_{1}^{*} \star P_{2}\right|^{2} \mathrm{~d}^{2} f}{\iint\left|P_{1} \star P_{1}^{*}+P_{2} \star P_{2}^{*}\right|^{2} \mathrm{~d}^{2} f}
$$

For unaberrated wavefronts $\left(\arg P_{1}=\arg P_{2}\right)$, the modulation of the fringes obtained with an unresolved source is equal to $1 / 2$ and the contrast to unity. Using the convolution theorem, it is straightforward to show that the numerator of Eq. (15) is equal to the overlap integral of the images formed by each sub-aperture in the absence of interference which is the cross-correlation of two Airy patterns evaluated at the separation $\boldsymbol{\alpha}_{1}-\boldsymbol{\alpha}_{2}$. The intensity function of the Airy pattern is the squared modulus of the amplitude expressed in Eq. (5) and is denoted by $I_{\mathrm{A}}$. Likewise, the denominator can be expressed in terms of overlap integrals. For the case of displaced images, one finally gets:

$$
\begin{aligned}
C & \propto \frac{2 \int I_{\mathrm{A}}\left(\boldsymbol{\rho}-\boldsymbol{\alpha}_{1}\right) I_{\mathrm{A}}\left(\boldsymbol{\rho}-\boldsymbol{\alpha}_{2}\right) \mathrm{d} \boldsymbol{\rho}}{2 \int I_{\mathrm{A}}(\boldsymbol{\rho})^{2} \mathrm{~d} \boldsymbol{\rho}+2 \int I_{\mathrm{A}}\left(\boldsymbol{\rho}-\boldsymbol{\alpha}_{1}\right) I_{\mathrm{A}}\left(\boldsymbol{\rho}-\boldsymbol{\alpha}_{2}\right) \mathrm{d} \boldsymbol{\rho}} \\
& =\frac{\int I_{\mathrm{A}}(\boldsymbol{\rho}) I_{\mathrm{A}}\left(\boldsymbol{\rho}+\boldsymbol{\alpha}_{1}-\boldsymbol{\alpha}_{2}\right) \mathrm{d} \boldsymbol{\rho}}{\int I_{\mathrm{A}}(\boldsymbol{\rho})^{2} \mathrm{~d} \boldsymbol{\rho}+\int I_{\mathrm{A}}(\boldsymbol{\rho}) I_{\mathrm{A}}\left(\boldsymbol{\rho}+\boldsymbol{\alpha}_{1}-\boldsymbol{\alpha}_{2}\right) \mathrm{d} \boldsymbol{\rho}}
\end{aligned}
$$

The distribution we obtained for various perturbations matches the curve given by Eq. (16), as demonstrated in Fig. 9. Using this interpolating function, we can obtain values accurate to within $2 \%$ of the true value. The importance of this derived relationship resides in the possibility to have a very quick access to the fringe visibility, i.e. to the signal-to-noise ratio, for any kind of perturbation applied to the optical system, by measuring the resulting displacement of the centroid of the spot produced by the perturbed part of the system. Moreover, by measuring the separation of the centroids in units of Airy disk diameter, the derived function can be generalized to Fizeau interferometers with arbitrary $B$ and $d$. 


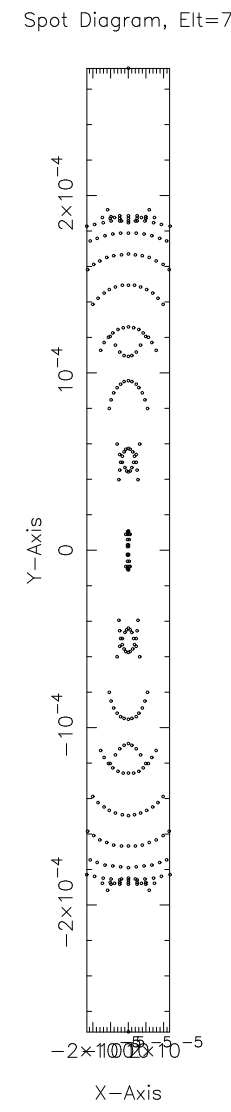

Fig. 8. Spot diagram given by COMP for the interferometer. Both axes are in units of centimeters. The geometrical spots overlap on the focal plane to within a small fraction of the Airy disk diameter. The overall size of the spot is nearly 4 $\mu \mathrm{m}$ (the size of the Airy disk is $38.75 \mu \mathrm{m}$ ). The numbers on the $x$-axis are $-210^{-5}, 0,210^{-5}$, thus representing a width of $0.4 \mu \mathrm{m}$

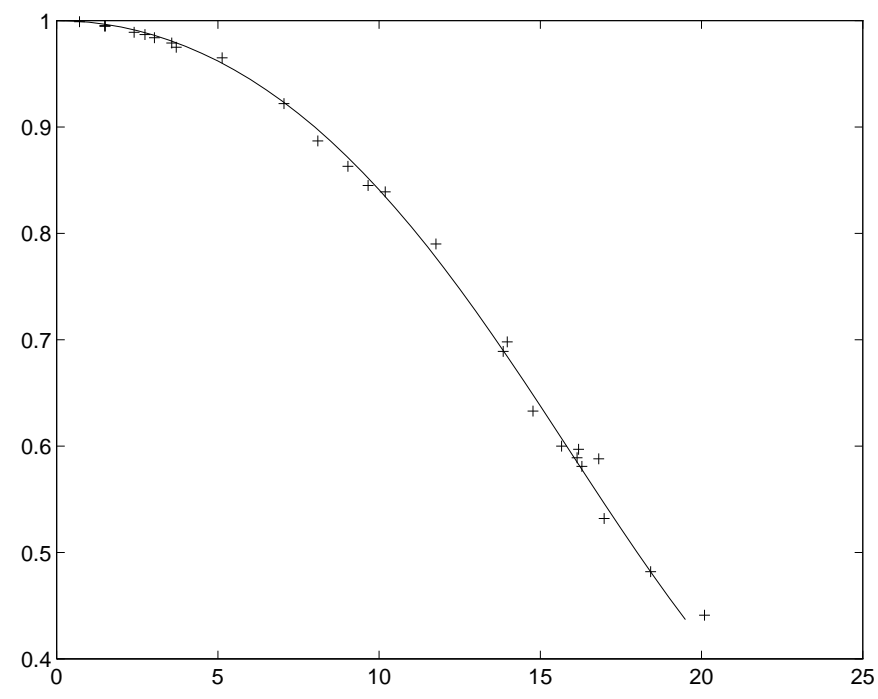

Fig. 9. Fitting function for the visibility vs. centroid separation relationship. The curve is derived from Eq. (16)
In this section, we have shown that the aberrations of the system only play a secondary role on the loss of visibility, compared to the relative tilts of the entrance sub-apertures. The main tolerances on these perturbations are, considering that a visibility of $\sim 0.75$ is acceptable, $\sim 0.5 \mu \mathrm{rad}$ for a tilt of a primary sub- aperture, $\sim 0.8 \mu \mathrm{rad}$ for a tilt of a secondary sub-aperture and $\geq 1 \mu \mathrm{rad}$ for a tilt of a tertiary sub-aperture. Tolerances on translations of the optics are less stringent, allowing motions of several microns along both the optical axis and the perpendicular ones. It is also possible to define a quality criterion in terms of the separation of the light centroids. If a visibility of 0.75 or higher is needed, our results show that the separation between the centroids of the images of each sub-aperture must remain smaller than $32 \%$ of the diameter of the corresponding theoretical Airy spot.

\subsection{Analysis of the distortion}

Distortion is the only aberration which does not affect image quality but only its position. With an astrometric

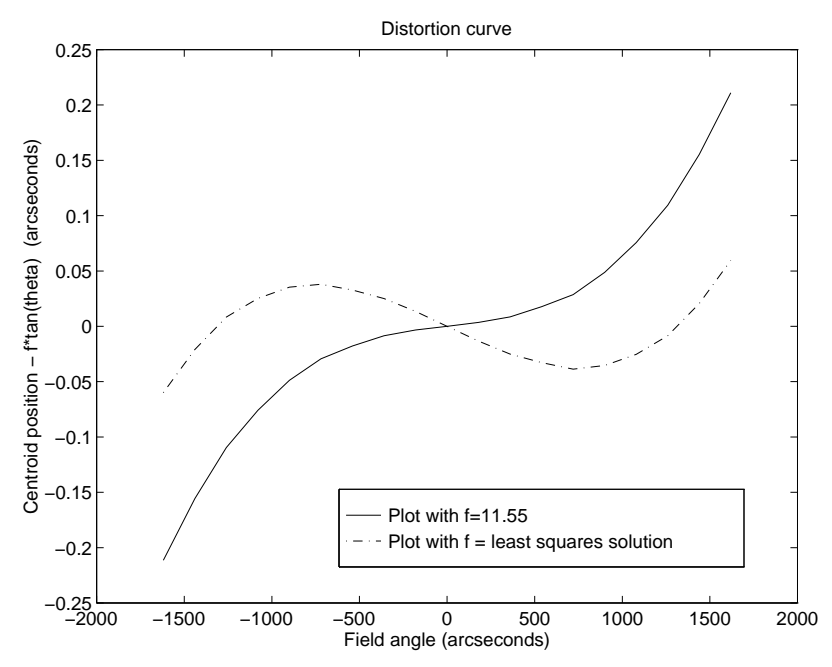

Fig. 10. Variations of the difference between the true centroid position and $f \times \tan \theta$ with respect to field angle

interferometer, we are interested in measuring very accurately the phase of the interference fringes. If they are displaced by unpredicted distortion, significant errors can occur. To avoid that, it is important to start with a distortion free or at least a low distortion instrument. Then, a precise model of the aberrations of the system would enable accurate fringe parameters measurements over the whole field-of-view. Our design was optimized for a wide field-of-view (0.9 degree) and is thus subject to distortion, since it scales as the cube of the field angle. Using COMP, its ray trace and diffraction features, we analyzed the distortion of the system for both the geometrical spot and the true diffracted image.

In a perfect system with no distortion, the centroid of the geometrical spot follows the variations of $f \times \tan \theta$ 

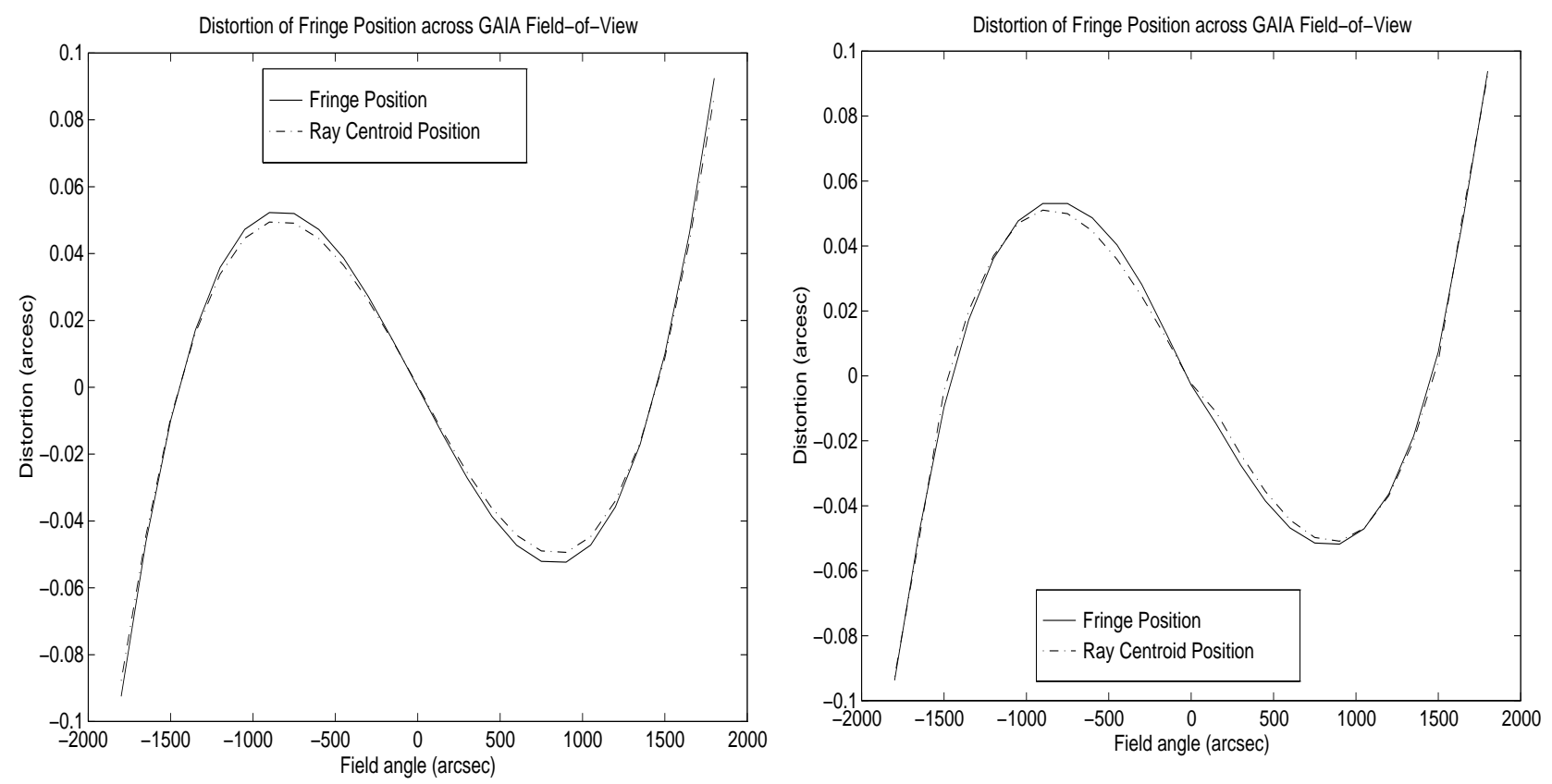

Fig. 11. Left a): Variations of the fringe position with respect to field angle. The amount of distortion at the edge of the field (0.45 degree) is $\sim 40$ mas. Right $\mathbf{b}$ ): Variations of the fringe position with respect to field angle with a primary sub-aperture tilted by $4 \mu \mathrm{rad}$

where $f$ is the paraxial focal length and $\theta$ the field angle. In a real system, the centroid's position is displaced by a third order term. We first plotted the difference between the actual centroid position and $f \times \tan \theta$ versus field angle. The relevant curve is the one for which $f$ is a least-squares fit of the system [centroid position versus $\tan \theta$ ]. These two curves are displayed in Fig. 10.

The second and most interesting step was to compute the position of the fringe pattern (the position of the central fringe for instance) and trace its variations across the whole field-of-view. Again, this could be done using COMP's full diffraction propagation. The variations of the fringe position with respect to field angle are plotted in Fig. 11a where it can be seen that there is $\sim 40$ mas of distortion at the edge of the field (0.45 degree). This corresponds to one fringe of displacement, an effect that would severely blur fringes if they were integrated across the focal plane, without caution.

As the optical elements of our system are perturbed, we compute the position of the central fringe, as explained previously. It seems that the amount of distortion is quite insensitive to these perturbations, at least for the order of magnitude we considered. For instance, Fig. 11b shows the same curve as Fig. 11a after tilting one of the primary subapertures by $4 \mu \mathrm{rad}$. It can be seen that the magnification of the fringe position is slightly different than before. The amount of distortion at the edge of the field is only $10 \%$ greater than before. Severe perturbations on one of the tertiary sub-apertures for instance, lead to similar results thus showing that the system does not seem to be subject to large variations of distortion within a wide range of perturbations.

\section{Conclusion}

The purpose of this paper was to present the modelling of a Fizeau interferometer and is intended as the first step towards an end-to-end modelling tool for an astrometric interferometer. We used an all-reflective compact threemirror telescope that we designed as well as a starting point for this study. It has a $\sim 0.9$ degree diffraction limited field-of-view and fits in the Ariane V payload shroud. The three hyperboloidal mirrors from which the apertures of the interferometers are cut have reasonable eccentricities and radii of curvature. The proposed configuration enables a precise compensation of the aberrations and would naturally require an accurate manufacturing of the optics.

We analyzed the performances of the instrument in terms of fringe visibility since it is related to the signalto-noise ratio. To do so, we chose a commonly used method to estimate the fringe visibility when the elements of the optical train of the interferometer are independently perturbed. We derived a numerical function describing the variations of the fringe visibility with the motion of the centroids of the images formed by each aperture of the interferometer. This relationship enables us to quickly describe the response of the instrument to perturbations, in terms of fringe visibility. We can define a quality criterion for the fringes in terms of separation of the centroids. We 
showed that when this separation remains smaller than $30 \%$ of the diameter of the theoretical unaberrated Airy pattern, the quasi-monochromatic visibility stays above 0.8. For polychromatic fringes $(\Delta \lambda=60 \mathrm{~nm})$, this value drops to 0.7 .

The instrument maintains good fringe visibility for perturbations corresponding to a temperature variation of $10 \mathrm{~K}$ over the whole structure made of low expansion material, and which are of the order of a few tenths of $\mu \mathrm{rad}$ for tips and tilts, making it quite robust to thermal and mechanical perturbations. It is very likely that a far more precise thermal control would be achieved in the final configuration, making the assumption on the order of magnitude of the perturbations quite pessimistic. It has also been shown that there is $\sim 40$ mas of distortion at the edge of the field and that distortion seems to be fairly insensitive to small perturbations of the system. A careful modelling of the aberrations and in particular distortion is thus needed so as to perform accurate fringe phase measurements over the whole field-of-view.

Acknowledgements. The authors wish to thank Lennart Lindegren and Michael Perryman for providing the subject of this study and the information needed to perform it, Jean Kovalevsky and Mike Shao for fruitful discussions and support. Many thanks again to Lennart Lindegren for his careful reading of the paper. The research described was partially performed at the Jet Propulsion Laboratory, California Institute of Technology, under a contract with the National Aeronautics and Space Administration. This work was partially completed while the first author was at the Jet Propulsion Laboratory. This research was supported by the Centre National d'tudes Spatiales and Matra Marconi Space.

\section{References}

Beckers J.M., Merkle F., 1991, High-Resolution Imaging by Interferometry II, ESO, Garching bei Munchen, Germany

Eisenberg S., Pearson T., 1987, Two-Mirror Three-Surface Telescope, Proc. SPIE 751, 24

Gelles R., 1973, Appl. Opt. 12, 935

Korsh D., 1972, Appl. Opt. 11, 2986

Korsh D., 1977, Appl. Opt. 16, 2074

Lindegren L., Perryman M.A.C., Bastian U., et al., 1994, Global Astrometric Interferometer for Astrophysics. In: Breckinridge J.B. (ed.), Proc. SPIE 2200, 599

Lindegren L., Perryman M.A.C., 1996, A\&AS (in press)

Perryman M.A.C., et al., 1992, A\&A 258, 1

Reasenberg R.D., Babcock R.W., Murison M.A., Noecker M.C., Phillips J.D., 1994, POINTS: an Astrometric Spacecraft with Multifarious Applications. In: Breckinridge J.B. (ed.), Proc. SPIE 2200, 2

Redding D., Needels L., Wallace K., et al., 1992, Controlled Optics Modelling Package User Manual, Version 1.0, JPL document D-9816

Robb P., 1978, Appl. Opt. 17, 2677

Roddier F., Léna P., 1984, J. Opt. 15, 171

Rumsey N.J., 1969a, A Compact Three-Reflection Astronomical Camera, Optical Instruments and Techniques. In: Dickson J.H. (ed.). Oriel Press, Newcastle upon Tyne, 514

Rumsey N.J., 1969b, Telescope System Utilizing Three Axially Aligned Substantially Hyperbolic Mirrors, U.S. Patent No. 3, 460, 886

Shao M., Colavita M., Staelin D.H., et al., 1987, AJ 93, 1280

Shao M., 1993, Orbiting Stellar Interferometer. In: Reasenberg R.D. (ed.), Proc. SPIE 1947, 89

Wetherell W., Rimmer M., 1972, Appl. Opt. 11, 2817 